\title{
Urinary Neutrophil-Gelatinase Associated Lipocalin is a more Prognostic Biomarker to Distinguish Antenatal Hydronephrosis in Neonates
}

\author{
Hamid Mohammadjafari ${ }^{1}$, Alireza Rafiei ${ }^{2^{*}}$, Mohammad Abedi ${ }^{3}$, Abdolrasul Aalaee ${ }^{4}$, Araz Mohammad Mirabi ${ }^{2}$, Ehsan Abedi ${ }^{4}$ \\ ${ }^{1}$ Department of Pediatrics, Faculty of Medicine, Mazandaran University of Medical Sciences, Sari, Iran. \\ ${ }^{2}$ Molecular and Cell Biology Research Center, Department of Immunology, Faculty of Medicine, Mazandaran University of Medical Sciences, \\ Sari, Iran. \\ ${ }^{3}$ Medical student, Faculty of Medicine, Mazandaran University of Medical Sciences, Sari, Iran. \\ ${ }^{4}$ Department of Radiology and Nuclear Medicine, Faculty of Medicine, Mazandaran University of Medical Sciences, Sari, Iran.
}

Received: 18 May 2013

Revised : 27 Jun 2013

Accepted: 5 Aug 2013

Corresponding Author:

Alireza Rafiei

Molecular and Cell Biology Research

Center, Department of Immunology,

Faculty of Medicine, KM 17

Khazarabad Road, Khazar Sq, Sari,

Iran,

Phon:+98-113543614

E-mail: Rafiei1710@gmail.com

\begin{abstract}
Background: Routine diagnostic methods of Vesicoureteral reflux (VUR) are invasive and can cause exposure to radiation and may increase risk of urinary tract infections. Therefore, introducing reliable, non invasive methods might be more interested in pediatric nephrology. The objective of this perospective case control study was to evaluate the prognostic value of urinary neutrophilgelatinase associated lipocalin (uNGAL) on antenatal hydronephrosis (AH) with and without VUR.

Materials and Methods: A total of 50 patients diagnosed with AH; $78 \%$ males with mean age 5.71 \pm 2.1 years, including $27 \mathrm{AH}$ with VUR and $23 \mathrm{AH}$ without VUR, and 19 normal healthy children; $78.9 \%$ males with mean age $5.63 \pm 1.89$ years, were enrolled in this study. Urinary NGAL levels were measured by enzyme linked immunosorbent assay (ELISA).

Results: There was a significant difference in UNGAL concentration between $\mathrm{AH}$ patients and controls $(0.80 \pm 0.26$ and $0.29 \pm 0.27 \mathrm{ng} / \mathrm{ml}, \mathrm{p}<0.0001)$. However, the levels of uNGAL was not significantly deviated between AH patients with VUR compared to those without VUR ( $0.84 \pm 0.34$ vs. $0.75 \pm 0.13$, $\mathrm{p}=0.419)$. Standardization of NGAL based on urinary creatinine ( $\mathrm{uNGAL} / \mathrm{uCr}$ ) showed a significantly difference between AH neonates with VUR compared to those without VUR $(2.43 \pm 1.61$ vs. $1.91 \pm 0.79, \mathrm{p}<0.0001)$. Receiver operator characteristic (ROC) analysis revealed higher prognostic power of uNGAL for identifying $\mathrm{AH}$ with a sensitivity; $95.7 \%$, and specificity; $84.2 \%$. Meanwhile, the levels of uNGAL or NGAL/uCr ratio did not correlate with reflux grade or laterality.
\end{abstract}

Conclusions: The urinary level of NGAL and NGAL/Cr ratio might be a surrogate non invasive, reliable tool to distinguish hydronephrosis.

Keywords: NGAL; Antenatal hyronephrosis; Vesicoureteral reflux; Neonate; Creatinine; Urine

Please cite this article as: Mohammadjafari H, Rafiei A, Abedi M, Aalaee A, Mirabi AM, Abedi E. Urinary NeutrophilGelatinase Associated Lipocalin is a more Prognostic Biomarker to Distinguish Antenatal Hydronephrosis in Neonates. Res Mol Med. 2013; 1 (2): 10-16

\section{Introduction}

Abnormalities of the urinary tract are reported to account for $30-50 \%$ of fetal anomalies (1). Of these anomalies, antenatal hydronephrosis including vesicoureteral reflux (VUR) has been reported in approximately $10 \%-20 \%(2-3)$. Because VUR is one of the major challenging in pediatrics field, which might be associated with some serious consequences such as reflux nephropathy (4), attendance to this 
renal abnormality is more important in all infants. On the other hand, VUR is a detrimental condition which may lead to scarring, urinary tract infection (UTI), and chronic kidney disease (5). So that the early identification of VUR in neonates with antenatal hydronephrosis is more need to prevent other severe complications in which may lead to kidney loss of function.

Nowadays, the most common diagnostic tool for VUR is a voiding cystourethrography (VCUG) or direct radioisotope cystography (DRNC) which uses for male and female children, respectively. Not only these methods are based on radioisotope exposure of each child at early age and its physician but it has demonstrated that they increased the risk of UTIs. These limitations are justifies the ongoing search for noninvasive reliable urinary biomarkers with the potential to aid early fast identification of kidney injury. We need to perform radiating and invasive tools for diagnosing VUR. Both voiding cystoureterography and isotope cystography initiated with catheterization a disturbing procedure and exposed patient to radiation (6). In recent years some effort made to replace other noninvasive ways for predicting VUR, urinary biomarkers level was the most known of them.

Neutrophil gelatinase-associated lipocalin (NGAL), a $25 \mathrm{kDa}$ protein of lipocalin family with iron carrier capacity, was originally identified in neutrophils, but it is also expressed at low constant rate in some cell types (7). NGAL, also known as lipocalin 2, involves in multiple processes such as innate immunity, apoptosis, and renal development. Accordingly, in healthy individuals, low levels of NGAL are detectable in the blood and urine (8). However, higher expression of NGAL in response to various pathological states, such as inflammation, infection, and acute kidney injury has been reported in kidney, liver and epithelial cells (9-10). Unlike creatinine, NGAL is specifically induced in damaged nephron epithelium. Therefore, NGAL is not only would be a more promising indicator of acute kidney failure but it has reported that it is associated with worsening of clinical outcomes (1112) . Interestingly, over expression of NGAL is more rapidly occurred than that creatinine following kidney injury in which leads to increase urinary and serum NGAL levels (13). Therefore, this cohort study was aimed to assess whether urinary NGAL (UNGAL) could be a fast and reliable biomarker for predicting VUR among patients with antenatal hydronephrosis.

\section{Materials and Methods}

The prospective cohort study was conducted on 50 antenatal hydronephrotic patients referred to Boali Sina Hospital in Sari, North of Iran during Jan 2012 to June 2013. Twenty healthy neonates who matched to the patients for sex, age, and delivery type were considered as controls. All aspects of the current study were approved by the Medical Research Ethics Committee at the Mandazaran University of Medical Sciences and conformed to the ethical guidelines set forth in the Declaration of Helsinki. Prior to enrollment, all patient parents were given an explanation of the nature of the study, and written informed consent was obtained. The only inclusion criterion was the diagnosis of prenatal hyronephrosis, defined as the presence of renal pelviectasis, evidence of dilatation of the anterior posterior pelvic diameter (APPD) $\geq 7 \mathrm{~mm}$ on third trimester of gestational period and APPD $\geq 5 \mathrm{~mm}$ for those in less than third trimester of pregnancy. The patients with renal cysts, duplex system, renal dysfunction, any history of urinary tract infection, or syndromes of other congenital anomalies known to be associated with VUR, in addition, those with suspected urinary tract obstruction were excluded from the study. Postnatal ultrasonography examination was undertaken at the first and 6 weeks of age for all neonates according to standard protocol. The postnatal ultrasonography was carried out by a single independent expert radiologist blinded to the results of the Voiding cystourethrography (VCUG). VCUG was considered for the neonates at first week for patients with bilateral hydronephrosis and at $6-8^{\text {th }}$ week for those with unilateral forms.

The reflux was graded as $\mathrm{I}$ to $\mathrm{V}$ on conventional VCUG and qualitatively as three level of mild, moderate and severe on DRNC. These two separate findings were matched. Accordingly, grade 1 and 2 of VUR were considered as mild, grade 3 as moderate and grades 4 and 5 as severe VUR. $99 \mathrm{~m}$ DTPA scan were undertaken for the patients with moderate and severe hydronephrosis at the second postnatal month. Based on imaging findings patients were classified in three groups; hydronephrotic neonates with VUR (AH+VUR), antenatal hydronephrosis and normal VCUG (AH without VUR), and healthy control (Control). Healthy controls were whom have a history of normal prenatal sonography and referred to our center for routine examinations.

\section{Urine creatinine and NGAL assay}

Fresh voided urine samples were obtained at the first weak prior to contrast studies. Samples were collected in sterile containers and immediately centrifuged and the supernatant frozen at $-70^{\circ} \mathrm{C}$ until 
required. Urine was tested for presence of blood or leucocytes using urine analysis strips and the samples containing blood or leucocytes were excluded from the study. NGAL was determined by enzyme-linked immunosorbent assay (ELISA) kits (GenWay, San Diego, CA). Sample preparation and assay procedure were followed according to the manufacturer's recommendation. The absorbance values for standards and samples were obtained at $450 \mathrm{~nm}$ and the standard curves constructed for each assay were compared and used to minimize inter assay variation. The concentrations were interpolated from standard curves expressed in $\mathrm{ng} / \mathrm{ml}$. The sensitivity of kits to detect NGAL was $0.01 \mathrm{ng} / \mathrm{ml}$. To avoid any bias, all samples were analyzed blind. All samples were run in duplicate with the appropriate standards on 96-well micro plates.

\section{Statistic analysis}

Categorical data were presented as percentages while non categorical variables were expressed as mean \pm standard deviation (SD). Statistical analysis of difference between groups was determined by $\mathrm{t}$ test, Fisher exact test for two groups' comparison, one -way analysis of variance (ANOVA) for three groups comparisons and chi square for comparison of qualitative data. A receiver operating characteristics (ROC) analysis was employed to calculate the area under the curve (AUC) to find the best cutoff values providing the highest diagnostic specificity followed by the best sensitivity.

\section{Results}

Characteristics of VUR patients

The baseline clinical features of the cohort are summarized in Table 1. There was no significant difference in age between studied groups $(p=0.761)$. In addition, although the male gender was more prominent in all three groups but there was no significant difference in sex distribution among study groups. The most of neonates fulfilled gestational period and this trend was observed in all three groups $(\mathrm{P}=0.327)$. Caesarian delivery was the major finding in all subjects and the type of delivery was not met significant difference between study groups. A second objective was to assess laterality of hydronephrosis in neonates with antenatal pelviectasis. Accordingly, the frequency of unilateral hydronephrosis was higher than bilateral types in antenatal hydronephrotics patients with and without VUR but we have not seen any significant differences between two groups $(p=0.362)$. In addition, the VUR was more frequently in mild and moderate than severe in $\mathrm{AH}$ with VUR patients.

Table 1. Demographic and clinical findings of patients with antenatal hydronephrosis with or without vesicourethral reflux.

\begin{tabular}{|c|c|c|c|c|}
\hline & $\begin{array}{l}\text { AH+VUR } \\
(\mathrm{n}=27)\end{array}$ & $\begin{array}{l}\text { AH without VUR } \\
(n=23)\end{array}$ & $\begin{array}{l}\text { Controls } \\
(\mathrm{n}=19)\end{array}$ & P-value \\
\hline Age-(day) & $5.93 \pm 2.1$ & $5.56 \pm 1.41$ & $5.63 \pm 1.89$ & 0.761 \\
\hline \multicolumn{5}{|l|}{ Sex } \\
\hline Male & $20(74.1)$ & $19(82.6)$ & $15(78.9)$ & \multirow[t]{2}{*}{0.764} \\
\hline Female & $7(25.9)$ & $4(17.4)$ & $4(21.1)$ & \\
\hline \multicolumn{5}{|l|}{ Gestational age } \\
\hline Term & $23(88.5)$ & $21(91.3)$ & $19(100)$ & \multirow[t]{2}{*}{0.327} \\
\hline Preterm & $3(11.5)$ & $2(8.7)$ & 0 & \\
\hline \multicolumn{5}{|l|}{ Type of delivery } \\
\hline Caesarian delivery & $22(91.7)$ & $20(87)$ & $17(89.5)$ & 0.72 \\
\hline \multicolumn{5}{|l|}{ laterality } \\
\hline Unilateral & $14(51.9)$ & $14(60.9)$ & - & \multirow[t]{2}{*}{$0.362 *$} \\
\hline Bilateral & $13(48.1)$ & $9(39.1)$ & - & \\
\hline \multicolumn{5}{|l|}{ Severity of reflux } \\
\hline Mild & $13(37.1)$ & - & - & \\
\hline Moderate & $13(37.1)$ & - & - & \\
\hline Severe & $9(25.7)$ & - & - & \\
\hline
\end{tabular}


Urine NGAL levels as an indicator of vesicoureteral reflux

In order to evaluate the prognostic value of uNGAL in children with VUR, the concentrations of NGAL was measured in three study groups. Noteworthy, creatinine is a routine indicator of kidney function, the level of this marker was also assessed in all groups. In addition, to normalize urine biomarkers for each sample, the urine levels of NGAL was standardized to the urine creatinine and expressed in $\mathrm{ng} / \mathrm{mg} \mathrm{uCr}$. As it was shown in Table 2, the levels of NGAL were significantly higher in AH patients with or without VUR compared to controls $(p<0.0001)$. Post Hoc multiple comparison analysis was revealed that the differences was significant only between patients and controls but not in patients with VUR and those without VUR $(p=0.419)$. After normalization of NGAL levels based on urine creatinine, in comparison to controls, the mean levels of NGAL/uCr ratio was significantly higher in AH patients with VUR than that AH without VUR $(\mathrm{p}<0.0001)$ (Table 2).

Table 2. Urine biomarker levels in antenatal hydronepherotic patients with or without VUR.

\begin{tabular}{lllll}
\hline & $\begin{array}{l}\text { AH+VUR } \\
(\mathbf{n}=27)\end{array}$ & $\begin{array}{l}\text { AHwithout } \\
\text { VUR(n=23) }\end{array}$ & $\begin{array}{l}\text { Controls } \\
(\mathbf{n}=19)\end{array}$ & P-value \\
\hline $\begin{array}{l}\text { Urine } \\
\text { creatinine }\end{array}$ & $0.423 \pm 0.18$ & $0.53 \pm 0.49$ & $0.53 \pm 0.32$ & 0.469 \\
NGAL & $0.84 \pm 0.34$ & $0.75 \pm 0.13$ & $0.29 \pm 0.28$ & $<0.0001$ \\
$\begin{array}{l}\text { NGAL/ } \\
\text { UCr }\end{array}$ & $2.43 \pm 1.61$ & $1.91 \pm 0.79$ & $0.67 \pm 0.67$ & $<0.0001$ \\
\hline
\end{tabular}

In the next step, we tempted to evaluate whether the levels of uNGAL could be a severity indicator of reflux. As it was shown in Table 3, although the levels of NGAL were higher in severe form of VUR compared to moderate or mild but these differences were not statistically significant $(\mathrm{p}=0.7)$.

Table 3. The effect of severity of VUR on urine NGAL levels.

\begin{tabular}{lll}
\hline & uNGAL $($ mean \pm SE $)$ & $\begin{array}{l}\text { uNGAL/Cr } \\
(\text { mean } \pm \text { SE })\end{array}$ \\
\hline Mild VUR & $0.83 \pm 04$ & $2.2 \pm 0.3$ \\
Moderate VUR & $0.79 \pm 0.1$ & $2.37 \pm 0.4$ \\
Severe VUR & $0.91 \pm 0.2$ & $2.36 \pm 0.8$ \\
P-value & 0.7 & 0.9 \\
\hline
\end{tabular}

This insignificant differences were also seen when the levels of NGAL normalized upon in urine creatinine $(\mathrm{p}=0.9)$.

In addition, the type of reflux was not significantly affected the production of NGAL ( $\mathrm{p}=0.596$,). On the other hand, the levels of NGAL was not differed in the patients with bilateral VUR and those with unilateral VUR $(0.8 \pm 0.2$ vs. $0.9 \pm 0.4 \mathrm{ng} / \mathrm{ml}$, $\mathrm{p}=0.596$ ).

\section{Sensitivity and Specificity in uNGAL biomarker}

In the final step, we assessed the anticipative power of uNGAL for identifying antenatal hydronephrosis. Figure 3 shows the ROC curve analysis of uNGAL to predict the VUR in patients with antenatal hydronephrosis. The area under the curve (AUC) was 0.537 with an optimal cutoff value of 0.725 $\mathrm{ng} / \mathrm{mL}$, sensitivity; $50 \%$ and specificity; $47.8 \%$.

In addition, when we standardized absolute uNGAL upon in urinary creatinine levels, the prediction levels of NGAL for VUR was not significantly changed in comparison to absolute NGAL levels. Accordingly, the sensitivity, specificity, and AUC for $\mathrm{uNGAL} / \mathrm{uCr}$ were $50 \%, 56.5 \%$ and 0.565 , respectively.

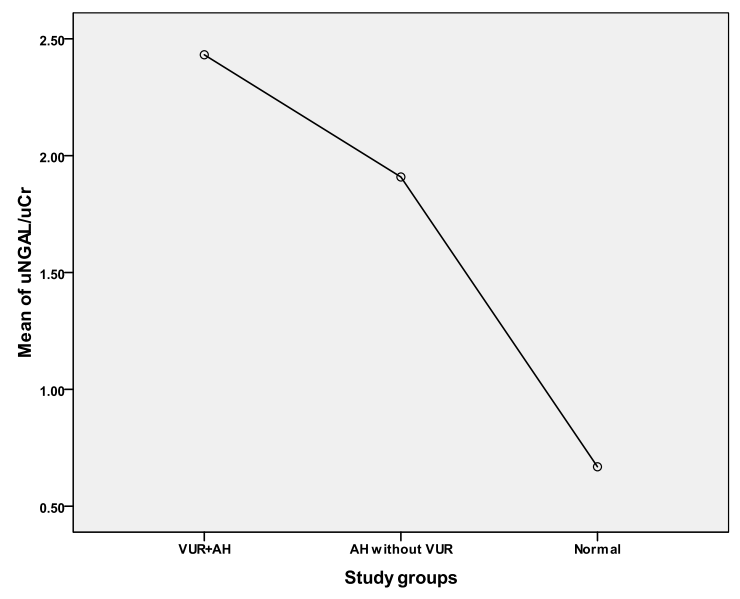

Figure 1. Mean plot urine $\mathrm{NGAL/uCr}$ ratio in antenatal hydronephrotics neonates with or without VUR.

For comparing hydronephrotic patients from normal neonates, the AUC, sensitivity, and specificity of urinary NGAL value of $0.565 \mathrm{mg} / \mathrm{ml}$ were 0.914 , $95.7 \%$ and $84.2 \%$, respectively (Table 4 ).

\section{Discussion}

vesicoureteral reflux is a recognized cause of antenatal hydronephrosis and is associated with significant morbidity (14). The main finding of this study was a significant increased in uNGAL levels in antenatal hydronephrotic patients compared to healthy controls. On the other hand, uNGAL would 
be more likely to be more sensitive tool to identify hydronephrosis in neonates (sensitivity, 95.7\%). It also has a reasonable specificity to differentiate antenatal hydronephrosis with specificity up to $84.2 \%$. Meanwhile, this biomarker was not shown a desirable sensitivity and specificity to differentiate vesicoureteral reflux in a background of antenatal hydronephrosis. The major source of NGAL is

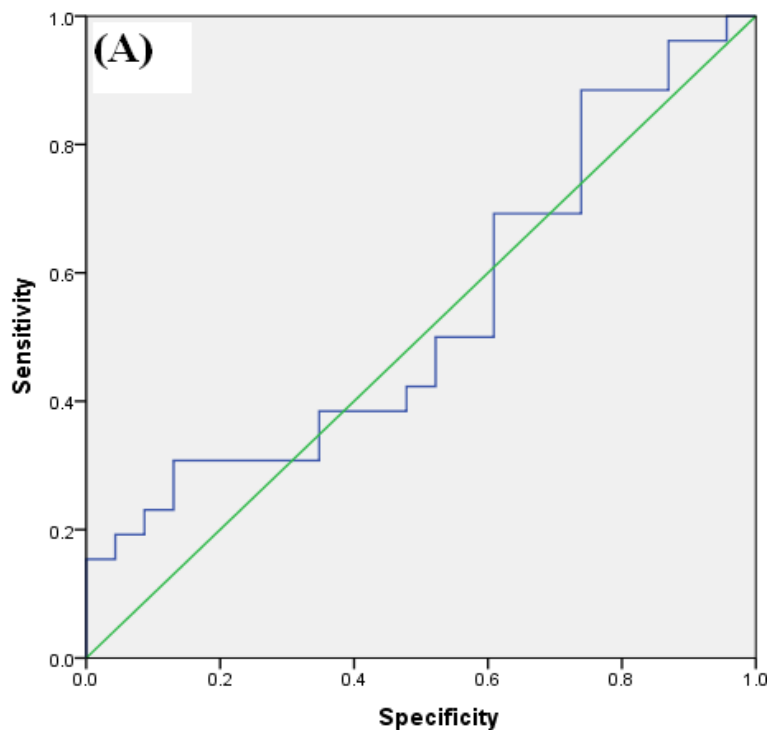

neutrophil which produce this natural immunity protein with antibacterial properties it in response to Gram negative bacterial infections (15-16). However, NGAL notably induce in some other conditions not necessarily associated with infective processes, such as inflammatory processes, cancers, and acute renal damage.

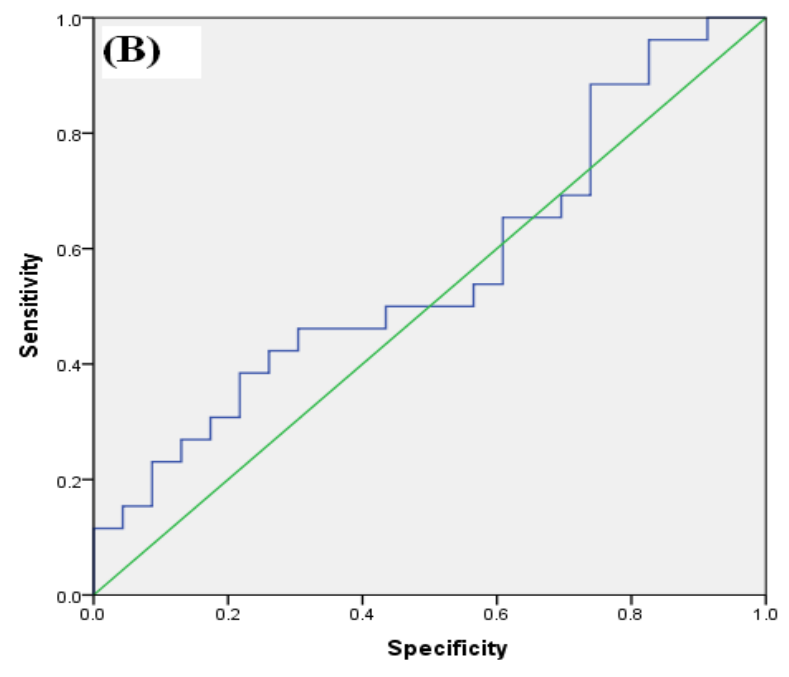

Figure 3. Receiver operating curve to identify VUR, (detect group1 from 2). (A) curve is for urinary NGAL [area under curve (AUC); 0.537 , SE: $0.84,95 \%$ CI: 0.372 to 0.702 )]. (B) curve shows urinary NGAL/uCr ratio [AUC; 0.565 , SE; $0.83,95 \%$ CI: 0.403 to 0.728 )].

VUR: Vesicoureteral reflux, SE: Sensitivity, CI: Confidence interval.

Otherwise, insignificant differences in uNGAL levels between antenatal hydronephrotic patients with VUR and those without VUR is accordance to an experimental ischemia-reperfusion study using aNGAL knockout mice that showed this protein is effectively able to prevent early damage, but probably did not promote the advance processes (16). The value of NGAL in pediatric field of medicine was most assessed in kidney injury states. Askenazi in 2 nested case-control studies on very low birth weight and normal weight neonates found that urinary level of NGAL was higher in neonates with acute kidney injury compared with normal ones (17-18).Recently, a fewinvestigations were performed to evaluate such levels in obstructive hydronephrotic children. Mia Gebauer Madsen et al., in a study on children suffered from ureteropelvic junction obstruction, evaluated NGAL levels in preoperative, perioperative, and postoperative periods and found a significant increased of NGAL in the perioperative urine compared to healthy controls. However, this surge of NGAL concentration was transient and its trend followed with an insignificant declined in 3and 12 month post operation (19). Increased levels compared to healthy controls is in perfect agreement with those observations in a prospective study conducted by Wasilewska et al., reporting significantly higher levels of uNGAL/Cr in children with congenital hydronephrosis caused by ureteropelvic junction obstruction (20). Recently, Cost et al., in a prospective cohort study in patients treated with unilateral dismembered pyeloplasty showed an increased level of bladder NGAL in children with ureteropelvic junction obstruction compared to controls (5). Interestingly, up regulation in NGAL production has been reported to be associated with worsening obstruction in patients with ureteropelvic junction obstruction (4). However, we didn't enrolled obstruction as an inclusion criterion but the urinary NGAL strict and standardized values were Significantlyhigher in hydronephrotic groups those normal neonates. We also calculated two cut off values of urinary NGAL concentration and NGAL-/uCr ratio with high sensitivity and specificity todistinguish hydronephrotic newborns from normal ones. 
Table 4. Sensitivity and specificity and of urinary levels of NGAL and NGAL/uCr ratio for comparing hydronephrotic patients (AH) with normal neonates and AH with VUR (AH+VUR) group compared with AH without VUR.

\begin{tabular}{|c|c|c|c|c|c|c|}
\hline \multirow[t]{2}{*}{ Marker } & \multicolumn{3}{|c|}{ AH vs. healthy controls } & \multicolumn{3}{|c|}{ AH+VUR vs. AH without VUR } \\
\hline & Level & Sensitivity & Specificity & Level & Sensitivity & Specificity \\
\hline Urinary NGAL & 0.5650 & 95.7 & 84.2 & 0.7225 & 50 & 47.8 \\
\hline Urinary NGAL/Cr （ng/mg) & 0.8291 & 91.3 & 73.7 & 2.0855 & 50 & 56.5 \\
\hline
\end{tabular}

The main aim of our study was the importance of NGAL biomarker for anticipating VUR in hydronephrotic neonates. We also calculated two cut off values of urinary NGAL concentration and

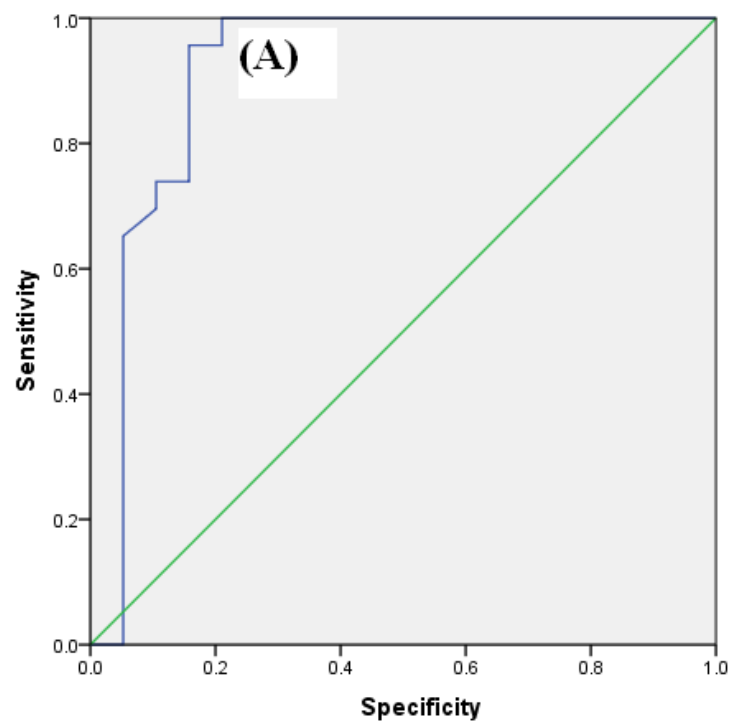

NGAL/uCr ratio with high sensitivity and specificity to distinguish hydronephrotic newborns from normal ones.

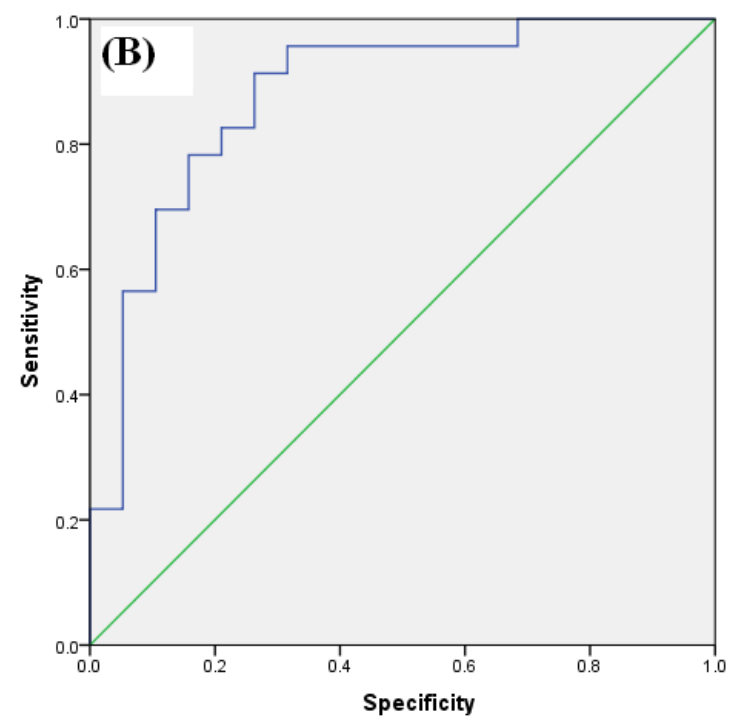

Figure 4. Receiver operating curve to detect AH, (detect group 2 from 3). (A) curve was shown for urinary NGAL (AUC; 0.914, SE 0.055, 95\% CI: 0.806 to 1.000 )]. (B) curve for urinary NGAL/Cr ratio (AUC; $0.879, \mathrm{SE} ; 0.055,95 \% \mathrm{CI} ; 0.770$ to 0.987 )].

VUR: Vesicoureteral reflux, SE: Sensitivity, CI: Confidence interval

The main aim of our study was the importance of NGAL biomarker for anticipating VUR in hydronephrotic neonates. We found that the urinary level of NGAL was not differed between patients with VUR and those without reflux but urinary NGAL/Cr ratio was significantly higher in neonates with VUR. In contrast to our finding, Ichino et al. demonstrated that in comparison to normal children, urinary NGAL levels were significantly increased in patients with VUR and renal scarring without evidence of UTI (21). However, these discrepancies might be explained by reasons such as; 1- presence of infection in which demonstrated that infection potentates NGAL expression through toll like receptor signaling (22), 2- age differences, accordingly, it has reported NGAL expression/ secretion has anage-dependent manner in which the most NGAL concentration is produced during the first year of age and then its level rapidly declined in normal children (21), 3- other sources for NGAL production except tubular cells (23-24). Taken to gather, although the levels of NGAL weren't differentiated VUR in antenatal hydronephrotic children but the normalized analyte based on $\mathrm{Cr}$ levels might be a prognostic non invasive tools to predict VUR. Therefore we cannot suggest uNGAL level for anticipating VUR but urinary $\mathrm{NGAL} / \mathrm{Cr}$ ratio is a relatively useful measure for this purpose.

\section{References}

1. Greig JD, Raine PA, Young DG, Azmy AF, MacKenzie JR, et al. Value of antenatal diagnosis of abnormalities of the urinary tract. BMJ. 1989; 298: 1417-9. PMID: 2502275

2. Gunn TR, Mora JD, Pease P. Antenatal diagnosis of urinary tract abnormalities by ultrasonography after 28 weeks' gestation: incidence and outcome. Am J Obstet Gynecol. 1995; 172: 479- 


\section{PMID: 7856673}

3. Mohammadjafari H, Alam A, Kosarian M, Mousavi SA, Kosarian S. Vesicoureteral reflux in neonates with hydronephrosis; Role of imaging tools. Iran J Pediatr. 2009; 19: 347-53.

4. Avner ED, Chavers B, Sullivan EK, Tejani A. Renal transplantation and chronic dialysis in children and adolescents: the 1993 annual report of the North American Pediatric Renal Transplant Cooperative Study. Pediatr Nephrol. 1995; 9: 61-73. PMID: 7742226

5. Sirin A, Emre S, Alpay H, Nayir A, Bilge I, Tanman F. Etiology of chronic renal failure in Turkish children. Pediatr Nephrol. 1995; 9: 549-52. PMID: 8580006

6. Mohammadjafari H, Mohammadjafari R. Postnatal management of prenatal hydronephrosis. J Clin Exc. 2013; 1: 63-80.

7. Kjeldsen L, Cowland JB, Borregaard N. Human neutrophil gelatinase-associated lipocalin and homologous proteins in rat and mouse. Biochim Biophys Acta. 2000; 1482: 272-83. PMID: 11058768

8. Singer E, Markó L, Paragas N, Barasch J, Dragun D, Müller DN, et al. Neutrophil gelatinase-associated lipocalin: pathophysiology and clinical applications. Acta Physiol (Oxf) 2013; 207: 663-72. PMID: 23375078

9. Mishra J, Ma Q, Prada A, Mitsnefes M, Zahedi K, Yang J, et al. Identification of neutrophil gelatinase-associated lipocalin as a novel early urinary biomarker for ischemic renal injury. J Am Soc Nephrol. 2003; 14 (10): 2534-43. PMID: 14514731

10. Goetz DH, Holmes MA, Borregaard N, Bluhm ME, Raymond $\mathrm{KN}$, Strong RK. The neutrophil lipocalin NGAL is a bacteriostatic agent that interferes with siderophore-mediated iron acquisition. Mol Cell. 2002; 10:1033-43. PMID: 12453412

11. Haase M, Devarajan P, Haase-Fielitz A, Bellomo R, et al. The outcome of neutrophil gelatinase-associated lipocalin-positive subclinical acute kidney injury: a multicenter pooled analysis of prospective studies. J Am Coll Cardiol. 2011; 57: 1752-61. PMID: 2151111

12. Wasilewska A, Taranta-Janusz K, Dębek W, Zoch-Zwierz W, Kuroczycka-Saniutycz E. KIM-1 and NGAL: new markers of obstructive nephropathy. Pediatr Nephrol. 2011; 26: 579-86. PMID: 21279810

13. Schmidt-Ott KM. Neutrophil gelatinase-associated lipocalin as a biomarker of acute kidney injury where do we stand today? Nephrol Dial Transplant. 2011; 26: 762-4. PMID: 21310736

14. Fasolato V, Poloniato A, Bianchi C, Spagnolo D, Valsecchi L, Ferrari A, Paesano P, et al. Feto-neonatal ultrasonography to detect renal abnormalities: evaluation of 1-year screening program. Am J Perinatol. 1998; 15: 161-4. PMID: 9572370.
15. Fjaertoft G, Foucard T, Xu S, Venge P. Human neutrophil lipocalin (HNL) as a diagnostic tool in children with acute infections: a study of the kinetics. Acta Paediatr. 2005; 94: 661-6. PMID: 16188765

16. Berger T, Togawa A, Duncan GS, Elia AJ, You-Ten A, Wakeham A, et al. Lipocalin 2-deficient mice exhibit increased sensitivity to Escherichia coli infection but not to ischemiareperfusion injury. Proc Natl Acad Sci USA. 2006; 103: 1834-9. PMID: 16446425

17. Askenazi DJ, Montesanti A, Hunley H, Koralkar R, Pawar P, Shuaib F, et al. Urine biomarkers predict acute kidney injury and mortality in very low birth weight infants. J Pediatr. 2011; 159: 907-12.e1. PMID: 21784446

18. Askenazi DJ, Koralkar R, Hundley HE, Montesanti A, Parwar $\mathrm{P}$, Sonjara $\mathrm{S}$, et al. Urine biomarkers predict acute kidney injury in newborns. J Pediatr. 2012; 161: 270-5.e1. PMID: 22424940

19. Madsen MG, Nørregaard R, Palmfeldt J, Olsen LH, Frøkiær J, Jørgensen TM. Urinary NGAL, cystatin C, $\beta 2$-microglobulin, and osteopontin significance in hydronephrotic children. Pediatr Nephrol. 2012; 27: 2099-106. PMID: 22710694

20. Wasilewska A, Taranta-Janusz K, Dębek W, Zoch-Zwierz W, Kuroczycka-Saniutycz E. KIM-1 and NGAL: new markers of obstructive nephropathy. Pediatr Nephrol. 2011; 26: 579-86. PMID: 21279810

21. Ichino M, Kusaka M, Kuroyanagi Y, Mori T, Morooka M, Sasaki $\mathrm{H}$, et al. Urinary neutrophil-gelatinase associated lipocalin is a potential noninvasive marker for renal scarring in patients with vesicoureteral reflux. J Urol. 2010; 183: 2001-7. PMID: 20303517

22. Yilmaz A, Sevketoglu E, Gedikbasi A, Karyagar S, Kiyak A, Mulazimoglu M, et al. Early prediction of urinary tract infection with urinary neutrophil gelatinase associated lipocalin. Pediatr Nephrol. 2009; 24: 238792. PMID: 19649660

23. Petrovic S, Bogavac-Stanojevic N, Peco-Antic A, Ivanisevic I, Kotur-Stevuljevic J, Paripovic D, et al .Clinical application neutrophil gelatinase-associated lipocalin and kidney injury molecule-1 as indicators of inflammation persistence and acute kidney injury in children with urinary tract infection. Biomed Res Int. 2013; 2013: 947157. PMID: 23936859

24. Mårtensson $\mathrm{J}, \mathrm{Xu} \mathrm{S}$, Bell $\mathrm{M}$, Martling $\mathrm{CR}$, Venge $\mathrm{P}$. Immunoassays distinguishing between HNL/NGAL released in urine from kidney epithelial cells and neutrophils. Clin Chim Acta. 2012; 413: 1661-7. PMID: 22609864 